\section{Monoclonal metastases}

Whereas molecular heterogeneity of tumors has been well documented, less well understood are the molecular underpinnings of metastases. Using high-resolution chromosome mapping tools, Bova and colleagues show that distant metastases of prostate cancer are derived from a single tumor cell. The researchers studied the molecular signature using comparative genomic hybridization and significance analysis of microarray data of 85 metastases from 29 patients who had died of metastatic prostate cancer. Both analyses showed clustering within a single individual, supporting the notion of a clonal origin of the metastases. In contrast, tumors taken from the same anatomical site in different individuals or from different racial groups did not cluster. This finding was upheld at the single gene level: the androgen receptor gene was amplified variously in 15 of 17 patients but uniformly within the tumors of an individual patient. Whereas some copy number changes are found throughout a patient's samples (omniclonal) and others are not (subclonal), the individuals showed a distinct pattern of copy number changes ('personality') that is stable at multiple sites in a patient, which in one case involved 17 sites. This study illuminates the genomic profile of lethal prostate cancer; further work will be needed to show whether a similar process is seen in other cancers. (Nat. Med. 15, 559-565, 2009)

\section{ZFNs target endogenous plant genes}

Progress in plant biotech has long been hampered by the lack of mutagenic and transgenic strategies to modify specific loci predictably. Zincfinger nucleases (ZFNs) — fusions of specific DNA-recognition modules with an endonuclease domain — have been used for targeted engineering of several eukaryotic genomes and were previously shown to modify reporter genes in plants by inducing a double-stranded break at their target locus. Two groups now show that ZFNs can modify endogenous plant genes. Working with tobacco, Townsend et al. demonstrate relatively high-frequency disruption of acetolactate synthase isoforms, which confers resistance to imidazolinone and sulphonylurea herbicides. Shukla et al. introduce a gene conferring herbicide tolerance into the maize gene that encodes the enzyme catalyzing the final step in the synthesis of phytate, an antinutritional component of animal feed that also contributes to environmental pollution. The ZFN-modified corn plants faithfully transmitted these genetic changes to the next generation, and no off-target $\mathrm{ZFN}$-induced changes to the genome were observed. Although both groups used herbicide-based screens, the frequencies of insertional inactivation should be adequate to use highthroughput sequencing to identify recombinants from a population of transformants. (Nature, published online April 29, 2009; doi:10.1038/ nature07845 and 10.1038/nature07992)

Written by Kathy Aschheim, Laura DeFrancesco, Markus Elsner, Peter Hare \& Craig Mak

\section{Neutralizing antibody on tap}

After 25 years of intensive HIV vaccine research, the successful induction of a broadly neutralizing antibody response remains elusive. Now, Johnson et al. show that a passive immunization strategy can protect against viral challenges, bypassing the need for elicitation of an adaptive immune response. As traditional passive immunization using antibody injection is impractical and prohibitively expensive for widespread use, Johnson et al. use an adeno-associated virus to deliver the gene encoding a known simian immunodeficiency virus (SIV)-neutralizing antibody to muscle cells of macaque monkeys. The myofiber cells in the muscle in turn synthesize antibodies and secrete them into the bloodstream. This approach maintains neutralizing antibody titers for at least 12 months. When challenged with SIV one month after immunization, none of the monkeys developed AIDS, and a majority showed no sign of infection. By contrast, four of six untreated monkeys died within 60 weeks. It is likely that this approach will find applications outside the HIV field, where more traditional vaccination strategies also fail to stimulate the production of neutralizing antibodies. (Nat. Med., published online May 17, 2009; doi:10.1038/nm.1967)

$M E$

\section{Rational peptide specificity}

Nature has evolved proteins that bind with precise affinity to specific interaction partners. In contrast, human-directed efforts to design peptides for research or therapeutic purposes have focused primarily on maximizing protein-binding affinity but not specificity. To address this problem, Grigoryan et al. devise a novel computational framework called CLASSY (cluster expansion and linear programming-based analysis of specificity and stability). This procedure optimizes a peptide sequence so as to maximize the thermodynamic energy gap between a desired interaction and off-target interactions. Simultaneously, it ensures that the peptide still binds its desired target as tightly as possible. CLASSY was used to design peptides that bind one of the 20 families of human bZIP transcription factors. Designed peptides were tested in vitro on a protein microarray for their affinities to a panel of bZIP fragments that represented all 20 families. In total, 8 bZIP families could be targeted specifically (target family bound more than all others), and 11 other families were bound with lower specificity. Going forward, CLASSY could be applied to design proteins such as zinc-finger, Src-homology 2 or PDZ domains or their ligands to engage in specific interactions. (Nature 458, 859-864, 2009)

$C M$

\section{Pathways to germ cells}

Cells that resemble germ cells have been generated in vitro from embryonic stem cells, but the process is poorly understood and differentiation efficiencies are low. To improve these methods, it will be important to unravel the molecular mechanisms of germ cell formation. A recent paper by Ohinata et al. takes a step in this direction. Using a serum-free and feeder-free system to culture early mouse embryos, the authors found that epiblast cells differentiate towards primordial germ cells (PGCs) in the presence of extraembryonic ectoderm and in the absence of visceral endoderm - but not vice versa. Bmp4, which is secreted by extraembryonic ectoderm, was sufficient to generate PGCs, whereas Cer1, produced by visceral endoderm, blocked this process. Another factor produced by extraembryonic ectoderm, Bmp8b, promoted differentiation to PGCs by suppressing the inhibitory effects of visceral endoderm. The in vitrogenerated PGCs could become functional sperm after maturation of the cells in vivo, either by transplanting gonads reconstructed with several thousand PGCs or by injecting PGCs into the seminiferous tubules. (Cell $137,571-584,2009)$ 